\section{O cuidado pré-natal em hospital universitário: uma avaliação de processo}

\author{
Prenatal care in an university hospital: \\ evaluating the process
}

\begin{abstract}
The objective of this study was to evaluate the prenatal care process referring to the Prenatal Assistance to Low Risk Pregnant Women Program of the University Hospital of Maringá, Paraná State, Brazil. It was made a selection of quality criteria which evaluate the precocity in the process and the adequacy in the number of consultation during prenatal period, measured by the Humanization Program of Prenatal and Birth of the Health Ministry and the adequacy on the use of the prenatal care. The study showed that $44.5 \%$ of pregnant women started late the prenatal care suggest of the low offer and search to better quality care, which is manifested by spontaneous transference of pregnant women to other services to the program. The prenatal consultations were confirmed and the results showed a mean of 9.8 consultations per pregnant woman - higher than the recommended national parameters. The indicators used and developed to the evaluation of process still present some obstacles to the organizational access and need to define strategies which guarantee this fundamental policy of the Brazilian Unified Health System.
\end{abstract}

Prenatal Care; Services Evaluation; Maternal Health Services
Elizabeth Eriko Ishida Nagahama 1 Silvia Maria Santiago 2

\section{Introdução}

A mortalidade materna é um importante indicador da realidade social de um país e de seu povo, bem como da determinação política de realizar ou não ações de saúde comunitária. Tanaka ${ }^{1}$ aponta que a magnitude do problema da morte materna no Brasil é reflexo da qualidade da assistência recebida pelas mulheres durante o ciclo gravídico puerperal e, principalmente, da desintegração dos serviços de atenção à saúde da gestante.

Para Grandi \& Sarasqueta 2, a atenção prénatal deve ter como objetivo a precocidade do ingresso, a periodicidade dos atendimentos e a captação das gestantes. O momento da primeira visita pré-natal, bem como a freqüência das visitas antenatais são usualmente referidos como possíveis elementos contribuintes na diminuição das taxas de morbimortalidade perinatal. O aumento no número de consultas de prénatal é inversamente proporcional a taxas de mecônio no líquido amniótico, de índices de Apgar menores que 7 no primeiro minuto e quinto minutos, de natimortalidade, bem como na incidência dos nascimentos pré-termo e de fetos de baixo peso ${ }^{3}$.

Dessa forma, a assistência pré-natal é reconhecida, pelo seu impacto e transcendência, como um dos componentes que contribuem para redução significativa dos coeficientes de mortalidade materna. 
Apesar da importância do cuidado pré-natal, pesquisa realizada em 22 capitais brasileiras em 2002 demonstrou que a assistência prénatal oferecida no país é desarticulada e parcial. Em seus estudos, Serruya 4 identificou que apenas 5\% das gestantes inscritas no Programa de Humanização do Pré-natal e Nascimento do Ministério da Saúde (PHPN/MS) receberam o conjunto de atendimentos previstos no programa, e $22,63 \%$ realizaram o número de consultas de pré-natal considerado como ideal. A autora ressaltou a necessidade de ampliar o acesso aos serviços de saúde e, principalmente, de oferecer serviços de qualidade no ciclo gravídico puerperal.

Para Donabedian 5, a qualidade na atenção à saúde apresenta dois componentes básicos: a definição e medida do estado de saúde e das mudanças ou diferenças deste estado; e a especificação do cuidado médico associado com qualquer resultado relativo a um estado de saúde. Segundo o referencial teórico sistêmico proposto pelo autor - a tríade estrutura, processo e resultado -, a avaliação do processo reflete exatamente a essência da qualidade da atenção à saúde e contribui para chamar a atenção sobre a necessidade de corrigir o curso do programa ou projeto ainda em andamento 6 .

Sendo assim, a aplicação de critérios de qualidade para avaliar o processo da assistência pré-natal proporciona identificar o desempenho do serviço e evidencia a qualidade da assistência, sendo esta uma das condições para garantir a efetividade dos cuidados à gestante.

O estudo objetivou avaliar o processo de atenção pré-natal referente à utilização do cuidado pré-natal do Programa Assistência Prénatal às Gestantes de Baixo Risco, Hospital Universitário de Maringá (PPNBR/HUM), implantado em 1996 no Ambulatório de Especialidades do HUM, Paraná. As características singulares desse programa referem-se ao caráter de ensino e do modelo assistencial proposto, no qual o profissional enfermeiro conduz a assistência pré-natal em conjunto com os profissionais da equipe multiprofissional composta por médico obstetra, nutricionista, assistente social, fisioterapeuta, psicóloga e discentes de enfermagem.

\section{Material e método}

O presente estudo utilizou dados da dissertação de mestrado Avaliação de um Programa de Assistência Pré-natal: a Questão da Resolutividade, na qual analisou-se indicadores de processo que traduzem a qualidade da aten- ção prestada pela equipe multiprofissional do PPNBR/HUM 7.

Realizou-se um estudo transversal do PPNBR/HUM, efetuado através da transcrição de 213 prontuários hospitalares de gestantes, ou seja, todas as mulheres atendidas no período de 1o de janeiro de1998 a 30 de março de 2001. Os dados foram coletados no período de abril a maio de 2002, e as variáveis de estudo foram: idade gestacional de início do pré-natal em semanas, número de consultas de pré-natal realizadas e idade gestacional de ocorrência do parto.

Os dados foram digitados e analisados no programa Excel. O projeto de pesquisa foi aprovado pelo Comitê de Ética em Pesquisa, e os aspectos éticos da pesquisa relativos à garantia de sigilo das informações foram seguidos para a realização do estudo.

Fekete 8 considera que a utilização do serviço de saúde representa o consumo real de seus serviços. Portanto, nesse estudo, a utilização do cuidado pré-natal correspondeu à seleção de critérios de qualidade que avaliaram a precocidade no ingresso pré-natal e a adequação no número de consultas. Esta foi realizada considerando dois parâmetros de julgamento: o Índice de Adequação da Utilização do Cuidado Pré-natal (Adequacy of Prenatal Care Utilization-APNCU) e os critérios recomendados no Componente I - incentivo à assistência prénatal - do PHPN/MS.

\section{Índice de Adequação da Utilização do Cuidado Pré-natal}

O primeiro critério de julgamento foi o APNCU, criado por Kotelchuck 9 em 1987. O índice APNCU caracteriza a adequação da utilização do cuidado pré-natal em duas dimensões independentes e distintas:

a) Adequação do início do cuidado pré-natal ou mês em que o cuidado pré-natal foi iniciado: considera que quanto mais precoce, mais adequado o cuidado pré-natal. Estabelece a distribuição da gestação em quatro grupos: meses 1 e 2; 3 e 4; 5 e 6; e 7 a 9. A adequação do início do cuidado pré-natal corresponde a: ótimo: 1 o ou 2o mês; adequado: 3o ou 4o mês; intermediário: 5o ou 6o mês; inadequado: 7o mês ou mais tarde, ou sem assistência pré-natal.

b) Adequação dos cuidados recebidos ou porcentagem de visitas recebidas ajustadas para o mês de início da assistência pré-natal e para a idade gestacional da ocorrência do parto. A medida da adequação dos cuidados recebidos corresponde à razão entre o número de visitas recebidas do número esperado de visitas. O nú- 
mero esperado de visitas é baseado no padrão de visitas pré-natais para gestações não complicadas recomendado pelo American College of Obstetricians and Gynecologistis (ACOG) 10: uma visita por mês até 28 semanas, duas visitas por mês até 36 semanas e uma visita por semana até o nascimento. A adequação dos cuidados recebidos (proporção do número de visitas recomendado pelo ACOG recebidas desde o início do cuidado pré-natal até o término da assistência pré-natal ou nascimento) corresponde a: ótimo: maior ou igual $110 \%$; adequado: 80-109\%; intermediário: 50-79\%; inadequado: menos que $50 \%$.

Dessa forma, o APNCU combina a adequação do início da assistência pré-natal (mês de início da assistência) e a adequação da utilização do cuidado pré-natal (número de consultas realizadas) como sendo: ótimo: cuidado prénatal iniciado até o 4 o mês e realização de $110 \%$ ou mais das visitas recomendadas; adequado: cuidado pré-natal iniciado até o 4 o mês e realização de $80-109 \%$ das visitas recomendadas; intermediário: cuidado pré-natal iniciado até o 4o mês e realização de 50-79\% das visitas recomendadas; inadequado: cuidado pré-natal iniciado após o 4o mês ou realização de menos que $50 \%$ das visitas recomendadas.

\section{Programa de Humanização}

no Pré-natal e Nascimento

O Programa de Humanização no Pré-natal e Nascimento (PHPN) foi instituído pela Portaria $n^{\circ} .569$ do MS, em 1o de junho de 2000, constituindo uma das diretrizes para reorientar o modelo de assistência à saúde da mulher. O Componente I do PHPN - "Incentivo à Assistência Pré-natal" - recomenda oito critérios mínimos de qualidade para o adequado acompanhamento pré-natal e assistência à puérpera. Entre esses, o município deve assegurar à gestante a realização da primeira consulta de pré-natal até o 4o mês da gestação e realizar, no mínimo, seis consultas de acompanhamento pré-natal 11.

Portanto, esses foram o segundo critério de julgamento para mensurar a utilização do cuidado pré-natal.

Ressalta-se que, para utilização desses dois critérios, foi considerada a idade gestacional do início da assistência pré-natal como sendo a identificada na consulta de triagem no prénatal de baixo risco do HUM, mesmo se a gestante já havia iniciado a assistência pré-natal em outra instituição, pelo fato de não ter sido possível identificar a idade gestacional de início da assistência pré-natal quando a gestante já havia iniciado o pré-natal em outra instituição.

\section{Resultados e discussão}

A Tabela 1 classifica e compara a adequação da utilização do cuidado pré-natal do PPNBR/ HUM considerando os dois critérios.

Quanto à idade gestacional de início do cuidado pré-natal, a Tabela 1 demonstra que, em relação ao critério APNCU, o cuidado foi considerado ótimo para $30,3 \%$ das gestantes; adequado para $25,2 \%$; intermediário para $42,0 \%$ e inadequado para 2,5\% das mulheres. Quando comparado ao índice do PHPN, a adequação da utilização do cuidado pré-natal foi de 55,5\%, e a inadequação, de 44,5\%.

Ao considerar-se o número de consultas de pré-natal, observou-se que, de acordo com o critério APNCU, o cuidado pré-natal foi ótimo para $10 \%$ das gestantes; adequado para $48,8 \%$; intermediário para $38,7 \%$ e não houve inadequação para esse índice. Por outro lado, conforme critérios do PHPN, o cuidado recebido foi classificado como adequado para $91,6 \%$ das mulheres e inadequado para $8,4 \%$.

Portanto, ao considerar-se o sumário do índice de adequação (APNCU e PHPN), a utiliza-

Tabela 1

Índice comparativo de adequação na utilização do cuidado pré-natal segundo critérios do Índice de Adequação da Utilização do Cuidado Pré-natal (APNCU) e Programa de Humanização no Pré-natal e Nascimento (PHPN).

\begin{tabular}{|c|c|c|c|c|}
\hline \multirow{2}{*}{ Critério de avaliação } & \multicolumn{2}{|c|}{ APNCU } & \multicolumn{2}{|c|}{ PHPN } \\
\hline & $\mathrm{n}$ & $\%$ & $\mathrm{n}$ & $\%$ \\
\hline \multicolumn{5}{|c|}{ Utilização do cuidado pré-natal } \\
\hline Ótimo & 36 & 30,3 & - & - \\
\hline Adequado & 30 & 25,2 & 66 & 55,5 \\
\hline Intermediário & 50 & 42,0 & - & - \\
\hline Inadequado & 3 & 2,5 & 53 & 44,5 \\
\hline \multicolumn{5}{|c|}{ Adequação dos cuidados recebidos } \\
\hline Ótimo & 12 & 10,0 & - & - \\
\hline Adequado & 58 & 48,8 & 109 & 91,6 \\
\hline Intermediário & 46 & 38,7 & - & - \\
\hline Inadequado & - & - & 10 & 8,4 \\
\hline Ignorado & 3 & 2,5 & - & - \\
\hline \multicolumn{5}{|c|}{$\begin{array}{l}\text { Sumário do índice de adequação } \\
\text { na utilização do cuidado pré-natal }\end{array}$} \\
\hline Ótimo & 5 & 4,2 & - & - \\
\hline Adequado & 39 & 32,8 & 66 & 55,5 \\
\hline Intermediário & 19 & 16,0 & - & - \\
\hline Inadequado & 53 & 44,5 & 53 & 44,5 \\
\hline Ignorado & 3 & 2,5 & - & - \\
\hline
\end{tabular}

Fonte: Nagahama 7. 
ção do cuidado pré-natal foi inadequado para 53 (44,5\%) gestantes. Esse resultado foi atribuído ao início tardio da assistência pré-natal (após o mês 4) e não ao número insuficiente de visitas, pois não houve inadequação dos cuidados recebidos (relativo ao número de consultas de pré-natal) ao utilizar-se o índice APNCU. Este mensura a adequação do cuidado pré-natal, ajustando o número de consultas de prénatal para a idade gestacional de início e do término da assistência (ocorrência do parto), o que não ocorre ao utilizar-se o índice do PHPN, que avalia como adequadas seis consultas de pré-natal, independente dos dois fatores considerados no índice APNCU.

Chama atenção, neste estudo, o fato de que, ao identificar a procedência das 53 mulheres que iniciaram tardiamente o pré-natal no HUM (após o mês 4), o início tardio da assistência, representada por 36 (68\%) gestantes, ocorreu devido à transferência espontânea de outro serviço - 78\% deslocaram-se do Posto de Saúde próximo à sua residência.

Tase 12 aponta a ocorrência de peregrinação das mulheres em busca de assistência prénatal, e Oliveira 13 ressalta, em seu trabalho, que os principais fatores que têm contribuído para a busca da gestante pelo cuidado pré-natal em outras instituições de saúde constituem a qualidade técnica e humana dos profissionais de saúde.

No Município de Maringá, dois estudos realizados em núcleos integrados de saúde demonstram o aumento expressivo da busca, pela população, de atendimento em serviços de saúde fora da sua área de abrangência 14,15.

Campos 16 afirma que a adscrição da clientela à determinada unidade de saúde mensura diretamente o grau de qualidade ou de resolutividade da mesma. Segundo o autor, existem aqueles a quem não é dada a opção e devem compulsoriamente dirigir-se aos únicos serviços que os podem atender. Se os que podem optar elegem determinado serviço, é porque o serviço escolhido deve ter, segundo a sua percepção, determinada qualidade ou resolutividade para superar as das outras possíveis opções.

Acrescenta-se a esse fato as características do hospital universitário - centro de atendimento médico de alta complexidade e tecnologia -, e a concepção que existe entre a população de que, quanto maior e mais complexo o estabelecimento, melhor o atendimento 17 .

Ao comparar a adequação na utilização do cuidado pré-natal nos anos de 1998/2001, pode-se inferir que o percentual de gestantes que obteve assistência considerada como adequa- da aumentou cerca de duas vezes nos anos 1999/2000, bem como houve diminuição no número de gestantes com assistência pré-natal classificada como intermediária e inadequada (Tabela 2). Pelo fato de essa classificação depender basicamente da idade gestacional de início da assistência pré-natal (quanto mais precoce, melhor o cuidado pré-natal), pode-se apontar que duas mudanças na rotina de atendimento influenciaram esse resultado. O primeiro referiu-se ao aumento do número de enfermeiros no programa a partir de 1999, que proporcionou ampliação do número de vagas para atendimento pré-natal e aumento da capacidade de oferta do serviço. Outro fator associou-se à implantação da rotina de triagem no ambulatório para encaminhamento da gestante ao ambulatório de gestação de baixo ou alto risco, o qual proporcionou agendamento de consulta de pré-natal de forma mais ágil.

Portanto, as referidas mudanças na rotina de atendimento possibilitaram o ingresso precoce no cuidado pré-natal.

Quando verificada a adequação da utilização do cuidado pré-natal para o grupo de mulheres que iniciou o pré-natal no HUM, ou seja, que não transferiram seu atendimento prénatal de outro serviço para o HUM, observouse que $32 \%$ das mulheres iniciaram tardiamente o acompanhamento pré-natal. Isso aponta que existiram dificuldades no ingresso precoce ao cuidado pré-natal no HUM.

Essa afirmação é respaldada pelo estudo de Scochi 18, realizado no Município de Maringá, o qual identificou que a municipalização propiciou maior acesso da população aos serviços de saúde, todavia esse processo não foi acompanhado do desenvolvimento de ações preventivas sistemáticas. A autora apontou que as mulheres estão estimuladas a fazer o pré-natal, mas, por vezes, o serviço de saúde não está apto a recebê-las.

Nesse sentido, reporta-se ao conceito de acessibilidade proposto por Fekete 8 , definido como sendo o grau de ajuste entre as características dos recursos de saúde e as da população, no processo de busca e obtenção de assistência à saúde. A autora compreende que a acessibilidade, enquanto componente do processo de avaliação, resulta de fatores de diversas dimensões, classificadas como de ordem geográfica, organizacional, sócio-cultural e econômica. Os dados obtidos no estudo indicaram que existem obstáculos no modo de organização dos recursos de assistência à saúde, ou seja, em sua acessibilidade organizacional.

Em relação ao número de consultas de prénatal, a Figura 1 aponta que o número de con- 
sultas apresentou a seguinte ordem decrescente de freqüência: 61 (51,3\%) gestantes realizaram dez ou mais consultas; 28 (23,5\%), de oito a nove consultas; 20 (16,8\%), de seis a sete consultas, e 10 (8,4\%), menos que seis consultas.

Ao comparar-se o número de consultas de pré-natal com parâmetros recomendados pela Organização Mundial da Saúde e MS, pode-se inferir que 91,6\% das mulheres tiveram atendimento considerado como adequado; se comparado aos parâmetros do programa Protegendo a Vida no Estado do Paraná, esse índice corresponde a 86,6\%. Esses índices foram superiores ao encontrado em estudos nas regiões Sul e Sudeste do Brasil, no qual observaram-se valores que variaram de $50 \%$ a $77,5 \% 19$.

Em Maringá, estudo de Scochi 18 evidenciou que $80 \%$ das gestantes realizaram mais de seis consultas de pré-natal, das quais, $38 \%$ ultrapassaram dez consultas, e Sapata 15 , ao avaliar programa de assistência ao pré-natal de baixo risco em núcleo integrado de saúde, apontou que as gestantes realizaram, em média, 6,6 consultas médicas.

Portanto, o número médio de consultas de pré-natal encontrado no presente estudo - 9,8 consultas por gestante - demonstrou ser superior aos parâmetros mínimos recomendados pelos órgãos nacionais e internacionais de saúde, aos relatados em outros estudos brasileiros e a pesquisas realizadas em serviços públicos de saúde do município.

Esse maior número de consultas poderia apontar para a medicalização da assistência. Contudo, como um dos objetivos do programa constitui o desenvolvimento de trabalho terapêutico que estimule a capacidade de autonomia das mulheres, esse indicador representa a possibilidade de desenvolver continuamente práticas educativas, o atendimento das necessidades de saúde individuais e maior duração da relação profissional - gestante.

\section{Conclusão}

O estudo sugere que os fatores que contribuíram para o início tardio da assistência pré-natal para $44,5 \%$ das gestantes foram os relacionados ao serviço: a busca por melhor qualidade no atendimento e a dificuldade para ingresso precoce no pré-natal do HUM. Observou-se ainda que, segundo o índice APNCU, não houve inadequação dos cuidados recebidos, indicando que as consultas de pré-natal foram garantidas para todas as gestantes inscritas no programa. Dessa forma, observou-se que a inadequação do cuidado foi atribuída ao início

\section{Tabela 2}

Sumário comparativo do índice de adequação na utilização do cuidado pré-natal segundo critério Índice de Adequação da Utilização do Cuidado Pré-natal entre os anos 1998/2001.

\begin{tabular}{lcccc}
\hline Sumário & \multicolumn{4}{c}{ Ano } \\
& $\begin{array}{c}1998 \\
(\%)\end{array}$ & $\begin{array}{c}1999 \\
(\%)\end{array}$ & $\begin{array}{c}2000 \\
(\%)\end{array}$ & $\begin{array}{c}2001 \\
(\%)\end{array}$ \\
\hline Adequado & 20,0 & 35,6 & 48,4 & 20,0 \\
Otimo & - & 6,7 & 6,4 & - \\
Intermediário & 20,0 & 15,7 & 9,9 & 40,0 \\
Inadequado & 60,0 & 42,2 & 40,0 & 40,0 \\
\hline
\end{tabular}

Fonte: Nagahama 7 .

Figura 1

Distribuição das gestantes segundo número de consultas com enfermeiro do Programa Assistência Pré-natal às Gestantes de Baixo Risco, Hospital Universitário de Maringá, Paraná, Brasil, 1998/2001.

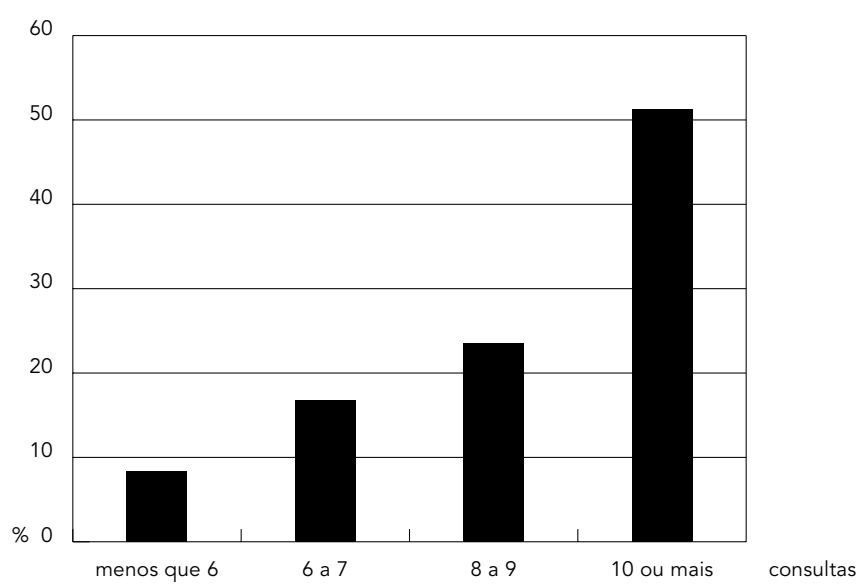

Fonte: Nagahama 7.

tardio da assistência pré-natal e não ao número insuficiente de visitas, pois o número médio de consultas foi superior aos parâmetros nacionais recomendados.

A aplicação de dois critérios de julgamento para mensurar a utilização do cuidado permitiu conclusões importantes, as quais não seriam conhecidas se fosse utilizado apenas o índice do PHPN do MS. Contudo, uma ressalva quanto ao uso do indicador APNCU referiu-se à utilização do sumário do índice de adequação na utilização do cuidado pré-natal, o qual 
não permitiu distinguir se a inadequação dos cuidados no pré-natal foi atribuída ao início tardio da assistência ou ao número insuficiente de visitas.

Kaestner 20 afirma que a utilização do cuidado pré-natal está relacionada a diversos fatores, seja os ligados à gestante ou ao serviço de saúde. Sendo o processo de trabalho envolvido na área da saúde complexo e, ao mesmo tempo, determinante nos resultados. A avaliação do processo reafirmou sua relevância ao apontar as diferentes dimensões intervenientes no cuidado pré-natal. A avaliação do pro-

\section{Resumo}

O objetivo do estudo foi avaliar o processo de atenção pré-natal referente à utilização do cuidado pré-natal do Programa Assistência Pré-natal às Gestantes de Baixo Risco do Hospital Universitário de Maringá, Paraná, Brasil. Compreendeu a seleção de critérios de qualidade que avaliaram a precocidade no ingresso $e$ a adequação do número de consultas de pré-natal, mensuradas conforme o Programa de Humanização do Pré-natal e Nascimento do Ministério da Saúde e Índice de Adequação da Utilização do Cuidado Prénatal. O estudo demonstrou que 44,5\% gestantes iniciaram tardiamente o pré-natal, o que pode sugerir uma oferta limitada de vagas e a busca por melhor qualidade na atenção, demonstrada pela transferência espontânea de gestantes de outros serviços para o programa. As consultas de pré-natal foram garantidas, sendo o número médio - 9,8 consultas por gestante-superior aos parâmetros nacionais recomendados. Os indicadores utilizados e desenvolvidos para a avaliação de processo identificaram que o serviço ainda apresenta obstáculos ao acesso organizacional, necessitando, assim, da definição de estratégias que garantam essa diretriz fundamental do SUS.

Cuidado Pré-natal; Avaliação dos Serviços; Serviços de Saúde Materna cesso de atenção pré-natal do programa permitiu inferir que o principal entrave para a utilização do serviço de saúde encontrou-se na forma de sua organização, que mostrou ser um obstáculo ao ingresso precoce da gestante ao cuidado.

Dessa forma, é necessário que os serviços de saúde estabeleçam estratégias que viabilizem o ingresso precoce das gestantes no prénatal, que garantam a oferta e acesso aos serviços e, principalmente, que promovam melhorias na qualidade da assistência prestada à mulher no ciclo gravídico puerperal.

\section{Colaboradores}

E. E. I. Nagahama redigiu a primeira versão e elaborou a versão final do texto. S. M. Santiago orientou e revisou o artigo final. 


\section{Referências}

1. Tanaka ACA. Mortalidade materna: reflexo da má qualidade e da desintegração dos serviços de saúde. Jornal da Rede Saúde 2000; 20:5-8.

2. Grandi C, Sarasqueta P. Control prenatal: evaluación de los requisitos básicos recomendados para disminuir el daño perinatal. J Pediatr (Rio J) 1997; 73:15-20.

3. Morais EN, Alflen TL, Spara P, Beitune PE. Momento e freqüência das visitas de pré-natal: repercussões sobre os nascimentos pré-termo. RBGO 1988; 20:25-32.

4. Serruya SJ. A experiência do Programa de Humanização no Pré-natal e Nascimento (PHPN) do Ministério da Saúde no Brasil [Tese de Doutorado]. Campinas: Faculdade de Ciências Médicas, Universidade Estadual de Campinas; 2003.

5. Donabedian A. The quality of medical care: a concept in search of a definition. J Farm Pract 1982; 9:975-92.

6. Gil CRR. Avaliação de projetos: análise do Projeto UNI - Londrina - A avaliação como instrumento da ação [Dissertação de Mestrado]. Londrina: Universidade Estadual de Londrina; 1995.

7. Nagahama EEI. Avaliação de um programa de assistência pré-natal: a questão da resolutividade [Dissertação de Mestrado]. Campinas: Faculdade de Ciências Médicas, Universidade Estadual de Campinas; 2003.

8. Fekete MC. Estudo da acessibilidade na avaliação dos serviços de saúde. In: Ministério da Saúde, organizador. Desenvolvimento gerencial de unidades básicas de saúde no distrito sanitário. Projeto Gerus. Brasília: Ministério da Saúde/Organização Pan-americana de Saúde; 1995. p. 177-84.

9. Kotelchuck M. An evaluation of the Kessner adequacy of prenatal care index and a proposed adequacy of prenatal care utilization index. Am J Public Health 1994; 84:1414-20.

10. American College of Obstetricians and Gynecologists. Standards for obstetric-gynecologic services. Washington DC: American College of Obstetricians and Gynecologists; 1985.

11. Ministério da Saúde. Programa de Humanização no Pré-natal e Nascimento: informações para gestores e técnicos. Brasília: Secretaria de Políticas de Saúde, Área Técnica da Saúde da Mulher; 2000.
12. Tase TH. Caracterização das mulheres atendidas em um hospital-escola referência para gestação de alto risco [Dissertação de Mestrado]. São Paulo: Escola de Enfermagem, Universidade de São Paulo; 2000.

13. Oliveira VM. Um lugar no cuidado pré-natal: possibilidades e opções das gestantes [Dissertação de Mestrado]. São Paulo: Escola de Enfermagem, Universidade de São Paulo; 2000.

14. Rainha ET. Análise dos encaminhamentos realizados pelo NIS II Vila Operária - Maringá - PR, para consulta especializada, em agosto de 1993 [Monografia de Especialização]. Maringá: Departamento de Enfermagem, Universidade Estadual de Maringá; 1994.

15. Sapata MPM. Avaliação da assistência ao pré-natal de baixo risco no NIS II Mandacarú - 1997, Maringá/PR [Monografia de Especialização]. Maringá: Departamento de Enfermagem, Universidade Estadual de Maringá; 1999.

16. Campos FE. Resolutividade: uma aproximação à avaliação qualitativa dos serviços de saúde [Tese de Doutorado]. Rio de Janeiro: Escola Nacional de Saúde Pública, Fundação Oswaldo Cruz; 1988.

17. Medici AC. Hospitais universitários: passado, presente e futuro. Rev Assoc Med Bras 2001; 47: 149-56.

18. Scochi MJ. Municipalização e avaliação de qualidade de serviços de saúde: uma análise localizada [Tese de Doutorado]. Rio de Janeiro: Escola Nacional de Saúde Pública, Fundação Oswaldo Cruz; 1996.

19. Neumann NA. Cobertura, qualidade e eqüidade da atenção ao pré-natal e ao parto, segundo a forma de pagamento destes serviços, em Criciúma SC [Tese de Doutorado]. São Paulo: Faculdade de Saúde Pública, Universidade de São Paulo; 2000.

20. Kaestner R. Health insurance the quantity and quality of prenatal care, and infant health. Inquiry 1999; 36:162-75.

Recebido em 26/Fev/2004

Versão final reapresentada em 02/Mai/2005

Aprovado em 19/Jul/2005 